\title{
Penser les droits catégoriels dans leur rapport à l'universalité
}

\author{
Danièle Lochak \\ Professeure émérite de l'Université Paris Ouest - Nanterre La Défense \\ Membre du CREDOF
}

\begin{abstract}
in Revue des Droits de l'Homme, n 3, juin 2013, « Les droits des femmes face à l'essor de l'intérêt de l'enfant », (en ligne : http://revdh.org/2-les-numeros-de-la-revue-des-droits-delhomme-2/numero-3-juin-2013/)
\end{abstract}

Droits des femmes, droits des enfants, droits des homosexuels, droits des travailleurs ou encore droits des minorités... L'ère des « droits [de l'Homme] catégoriels » aurait-elle succédé à l'ère des «droits [de l'Homme] universels »?

Parler de «droits catégoriels », n'est-ce pas postuler l'existence de droits qui ne seraient plus revendiqués par tous ni applicables à tous mais seulement à des catégories d'individus, sonnant ainsi le glas de l'universalité des droits de l'Homme?

Disons-le d'emblée : ni ce constat ni ce postulat, formulés à dessein sur un mode hypothétique, ne nous paraissent correspondre à une réalité qui est beaucoup plus nuancée. D'abord, l'histoire des droits de l'Homme a été jalonnée d'une série d'étapes qui témoignent d'un mouvement dialectique plutôt que d'une évolution linéaire, faisant alterner formulations universalistes et formulations spécifiques sans qu'on puisse assimiler les secondes à un recul des droits. Ensuite et surtout, l'idée d'un antagonisme de principe entre droits « universels » et droits « catégoriels » ne résiste pas à une analyse un peu poussée de la substance de ces droits dits « catégoriels 》 - au demeurant très hétérogènes et dont certains ne sont que la simple déclinaison concrète des droits universels - ni à la mise en lumière de leur fonction, la prise en compte des particularismes s'avérant parfois être la condition d'une universalité véritable.

\section{Retour sur l'histoire : un mouvement dialectique}

Si on tente de retracer la généalogie des droits de l'Homme, on trouve d'abord des textes qui consacrent des droits que l'on peut considérer comme catégoriels. C'est évident pour la Magna Carta de 1215 qui énumère les privilèges accordés à l'Église d'Angleterre, à la cité de Londres, aux marchands, aux seigneurs féodaux. Ça l'est aussi, quoique différemment, pour les textes anglais ultérieurs comme la Petition of Rights (1628) ou le Bill of Rights (1689) : contrairement aux déclarations de la fin du XVIII ${ }^{\mathrm{e}}$ siècle, ils n'entendent pas proclamer des principes abstraits et universellement valables mais remédier à des abus précis et garantir les libertés des Anglais ici et maintenant.

Avec la Déclaration des droits de l'Homme de 1789, la perspective change radicalement. La Déclaration de 1789 proclame des droits qui, inhérents à la nature humaine, sont nécessairement universels et valent pour tous les Hommes de tous les pays et de toutes les époques. L'universalité des droits proclamés découle aussi de ce que la Déclaration s'adresse à des sujets abstraits, à des individus dont les différences ou les appartenances n'ont pas à être prises en compte. C'est ainsi qu'il faut entendre la proclamation qui affirme solennellement que « la loi doit être la même pour tous » et qui vise avant tout à rappeler que tous les individus, égaux par essence, doivent être traités de façon identique.

Les différences, les particularismes de groupe sont donc gommés au nom à la fois de l'unité de la nature humaine et de l'égalité de tous les êtres humains. 
Cette conception universaliste, étroitement liée à la philosophie du droit naturel et qui inspire aussi, quoique de façon moins affirmée, les déclarations américaines ${ }^{1}$, ne survivra pas à la Révolution : avec la référence au droit naturel disparaît la vocation à l'universalité : ce sont au mieux les droits des Français que les constitutions ultérieures - qu'elles soient monarchiques, impériales ou républicaines - se contentent d'exposer.

Lorsque la préoccupation pour les droits de l'Homme ressurgit, en France, en 1848, elle s'exprime en des termes sensiblement différents. La constitution de la Seconde République parle des rapports entre patron et ouvrier, de l'assistance aux enfants abandonnés, elle évoque les infirmes, les vieillards, les chômeurs sans ressources... La reconnaissance des droits économiques et sociaux favorise, même si elle n'en est pas le seul facteur, une mutation dans la façon de penser les droits de l'Homme. Les titulaires de ces droits ne sont plus des hommes abstraits, ce sont des hommes concrets, saisis dans leur singularité de travailleurs ou de chômeurs, de malades ou de bien portants, de femmes ou d'enfants... Les droits de l'Homme ne visent plus l'Homme pris dans son essence et sa totalité, mais des catégories d'êtres humains ; ils ne postulent plus une nature humaine éternelle et immuable puisqu'ils prennent au contraire en considération les besoins d'individus situés dans un environnement social éminemment variable.

Plus récemment, un autre facteur est venu renforcer le développement de droits en apparence « catégoriels ». On vise ici la tendance contemporaine des mouvements contestataires à emprunter le langage des droits de l'Homme et à énoncer leurs revendications en termes de droits subjectifs : les groupes féministes, les mouvements de défense des chômeurs, des homosexuels, des prisonniers - ont placé leurs luttes sur le terrain du droit, réclamant l'inscription dans la loi des droits dont ils revendiquent le respect. Ce phénomène concourt à l'impression d'un foisonnement de droits catégoriels - sans qu'on doive pour autant en déduire, comme on le dira plus loin, une remise en cause des droits universels.

L'histoire de la protection internationale des droits de l'Homme est marquée par ce même mouvement dialectique. Les premières initiatives de la communauté internationale ne visaient pas à protéger les droits de l'Homme en général, mais des catégories de personnes particulièrement vulnérables, à l'image de la première Convention de Genève signée en 1864 sur la protection des blessés en temps de guerre, qui annonce le développement futur du droit international humanitaire. Dans l'entre-deux guerres, la Société des Nations reçoit la mission de veiller au sort des populations des pays sous mandat et de contrôler l'application des traités concernant les minorités nationales ; elle prend également des initiatives pour améliorer le sort des réfugiés. Parallèlement, sous l'égide de l'Organisation internationale du travail (OIT), créée en 1919, sont élaborées les premières conventions visant la protection des travailleurs.

La Déclaration universelle de 1948, point de départ du développement de la protection internationale des droits de l'Homme, marque une étape nouvelle. Rompant avec la démarche antérieure, elle entend protéger tous les droits de tous les êtres humains sans distinction, dans tous les pays, quels que soient leur sexe, leur origine, leur condition sociale, leur religion, leur langage et leurs convictions politiques, quels que soient les groupes - famille, cité, profession, confession, nation...- auxquels ils peuvent par ailleurs appartenir. Cette visée universelle se traduit dans les formules utilisées : " tous les êtres humains », « toute personne », " tout individu », « chacun », « tous » ou « quiconque ».

Si les deux Pactes de 1966 qui visent à mettre en œuvre la Déclaration universelle se situent dans cette même perspective, les dizaines de conventions conclues après 1945 sous l'égide de l'ONU ou de ses institutions spécialisées visent en revanche soit des violations spécifiques et particulièrement graves des droits de l'Homme (le génocide, la torture, les dispari-

${ }^{1}$ La première et la plus célèbre est la Déclaration des droits de l'État de Virginie de 1776, dont l'intitulé exact est : « Déclaration des droits qui doivent nous appartenir, à nous et à notre postérité » [souligné par nous]. 
tions forcées...), soit des catégories de personnes qui risquent plus que d'autres d'être victimes de discriminations ou d'atteintes à leurs droits fondamentaux, telles la Convention de Genève sur les réfugiés de 1951, les deux Conventions de 1965 et 1979 sur l'élimination de toutes les formes de discrimination raciale et sur l'élimination de toutes les formes de discrimination à l'égard des femmes, ou encore la Convention relative aux droits de l'enfant de 1989.

Cette évolution se repère également en ce qui concerne la protection internationale des minorités. Au sortir de la Première Guerre mondiale, les traités de paix et les traités de minorités contiennent des clauses qui reconnaissent à celles-ci, outre le droit à l'égalité civile et politique, une série de droits spécifiques tels que la liberté religieuse, le libre usage de la langue maternelle, la liberté d'enseignement, parfois l'autonomie locale. Dans les traités de paix signés après 1945, en revanche, les principales obligations faites aux États visent à assurer aux membres des minorités la jouissance des droits de l'Homme, sans distinction de race, de langue ou de religion.

De façon générale, la question des minorités est éclipsée au lendemain de la Seconde Guerre mondiale, où l'on éprouve davantage le besoin d'insister sur ce qui rapproche les hommes que sur ce qui les sépare, par le souci de mettre en place un système de protection des droits de l'Homme sur une base universelle. La Déclaration universelle de 1948 est donc muette sur la question des minorités et se borne à affirmer un principe - universel - de non discrimination et à interdire toute distinction, notamment de race, de couleur, de langue, de religion, d'origine ou de naissance. Le Pacte relatif aux droits civils et politiques de 1966 contient simplement un article 27 qui interdit aux États, là où il existe des minorités ethniques, religieuses ou linguistiques, de priver les membres de ces minorités « du droit d'avoir, en commun avec les autres membres de leur groupe, leur propre vie culturelle, de professer et de pratiquer leur propre religion, ou d'employer leur propre langue $»$.

C'est plus tard, avec l'effondrement des régimes communistes et la multiplication des conflits ethniques qui l'accompagne que va émerger à nouveau le problème des minorités au niveau international. Après une longue gestation, la « Déclaration des droits des personnes appartenant à des minorités nationales, ethniques, religieuses et linguistiques » est votée en 1992 par l'Assemblée générale des Nations unies, traduisant la prise de conscience que la reconnaissance des droits des minorités fait partie intégrante du respect des droits de l'Homme.

Parler d'évolution dialectique, c'est laisser entendre que si, historiquement, le passage de la protection de droits catégoriels à la reconnaissance de droits universellement valables a représenté un progrès dans la promotion des droits de l'Homme, la reformulation de ces droits pour prendre en compte les besoins ou les caractéristiques spécifiques de catégories d'êtres humains, sous des formes au demeurant très hétérogènes, ne peut être assimilée à une régression ou un abandon de l'ambition universaliste. C'est ce que nous nous proposons de démontrer.

\section{Un antagonisme plus apparent que réel}

Les droits catégoriels sont antagoniques avec les droits universels s'ils conduisent à réserver aux membres d'un groupe des droits dont les autres ne peuvent pas se prévaloir. Ce type de droits catégoriels a existé dans le passé sous la forme de privilèges réservés aux «puissants »: suffrage censitaire, droits de l'époux dans le Code civil, droits des Européens dans les colonies... Ce n'est évidemment plus le cas aujourd'hui. Reste que l'expression " droits catégoriels ", loin de correspondre à une notion uniforme, recouvre des réalités très diverses. Nous proposons ainsi de faire une distinction entre les droits catégoriels qui restent universels 
malgré leur formulation concrète, les droits catégoriels destinés à rendre plus effective l'universalité des droits, les droits effectivement catégoriels car reconnus de façon spécifique à des groupes qui réclament la reconnaissance de leur identité propre.

\section{Des droits catégoriels universels}

Les personnes auxquelles se réfère le Préambule de 1946 - les « hommes persécutés » qui ont droit d'asile sur le territoire de la République, les travailleurs, les familles, les enfants, les femmes en tant que mères... - comme avant lui la constitution de 1848 parlait des infirmes, des vieillards ou des chômeurs sans ressources, sont certes saisies dans leur singularité de travailleurs, de chômeurs, d'enfants, de vieillards, de persécutés... Mais les droits ainsi énoncés n'en restent pas moins universels. Reconnaître les droits des travailleurs, des chômeurs, des jeunes, des personnes âgées, des handicapés, des malades, des mères, c'est reconnaître des droits à tous les êtres humains, à l'ensemble des individus qui composent la société, mais saisis dans l'une de leurs qualités - temporaire ou contingente. L'énumération est plus englobante qu'excluante. Il en est de même lorsque le Pacte international sur les droits économiques, sociaux et culturels prévoit que tous les travailleurs doivent obtenir un salaire équitable qui leur procure une existence décente ainsi qu'à leur famille. La mutation dans la formulation des droits ne remet pas en cause leur universalité, même si on ne peut plus les rattacher à une " nature humaine » intemporelle et identiquement présente chez tous les Hommes.

Notons par ailleurs que des revendications exprimées sur un mode apparemment catégoriel ne visent souvent qu'à réclamer l'application réellement universelle des droits revendiqués. Ainsi, défendre les droits des femmes, c'est demander qu'elles jouissent de façon générale de droits identiques à ceux des hommes ${ }^{2}$; défendre les " droits des homosexuels », c'est demander qu'ils bénéficient des mêmes droits que le reste de la population - majoritairement hétérosexuelle - et non pas exiger qu'on leur accorde des droits spécifiques ; défendre les droits des étrangers, c'est réclamer, sinon idéalement l'égalité des droits, pour l'instant hors de portée, du moins un rapprochement de leur condition avec celle des nationaux; défendre les droits des prisonniers, c'est demander la mise en place d'un système plus respectueux de leurs droits fondamentaux que le régime d'exception auxquels ils sont soumis.

\section{Des droits catégoriels destinés à rendre effectifs les droits universels}

Un mode d'énonciation "catégoriel » apparaît dans certains cas comme une façon de prendre en compte la vulnérabilité de certains groupes dans le but de garantir l'effectivité véritable des droits proclamés comme universels sur une base d'égalité : c'est le cas des législations antidiscriminatoires qui désignent, en vue de leur accorder une protection spécifique, les catégories de personnes les plus exposées à la discrimination : femmes, Noirs, Arabes, homosexuels, malades...

C'est le cas aussi des dispositions conventionnelles ou législatives édictées dans un but de protection. Ainsi, la reconnaissance des droits de l'enfant par la Convention de 1989 est une façon de prendre acte de leur vulnérabilité particulière pour réaffirmer, d'une part, qu'ils jouissent de l'intégralité des droits de l'Homme (droit au respect de la vie privée et familiale, droit à un nom et à une nationalité, liberté de pensée et de religion, droit à la santé, droit à l'éducation...) et, de l'autre, qu'on doit leur accorder la protection spécifique dont ils ont besoin en raison de leur minorité (protection contre les violences, notamment sexuelles, les mauvais traitements, l'exploitation...). De même, la prise en compte des contraintes résultant

\footnotetext{
2 Même un droit apparemment aussi spécifique que le « droit à l'avortement » n'est à notre sens que la déclinaison de droits universels : le droit de disposer de son corps et le droit au respect de sa vie privée qui implique de pouvoir décider librement d'avoir ou non des enfants.
} 
pour les femmes de la gestation est nécessaire pour leur assurer l'égalité des droits, notamment dans la sphère professionnelle ${ }^{3}$.

La même remarque vaut pour la Convention sur l'élimination de toutes les formes de discrimination à l'égard des femmes de 1979 et la Convention sur toutes les formes de discrimination raciale de 1965 qui ont pour finalité, comme le rappelle leur intitulé, de permettre aux femmes, dans un cas, aux victimes désignées de la discrimination raciale, de l'autre, d'exercer effectivement l'intégralité des droits de l'Homme tels qu'ils sont énumérés dans la Déclaration universelle de 1948.

Ces deux Conventions autorisent les États à prendre des «mesures spéciales ", conçues comme des mesures de rattrapage, « visant à accélérer l'instauration d'une égalité de fait entre les hommes et les femmes » ou « à assurer comme il convient le progrès de certains groupes raciaux ou ethniques ou d'individus ayant besoin de la protection qui peut être nécessaire pour leur garantir la jouissance et l'exercice des droits de l'homme et des libertés fondamentales dans des conditions d'égalité ». Des dispositions analogues existent dans le droit de l'Union européenne : au départ, il s'est agi d'assurer aux femmes l' " égalité des chances ", au-delà de la seule « égalité de traitement »; depuis l'entrée en vigueur du traité d'Amsterdam la mise en place d' " actions positives » ou de « mesures spécifiques » est prévue par les directives adoptées dans le cadre de la lutte contre les discriminations afin de prévenir ou compenser des désavantages liés non seulement au sexe mais aussi à la race ou à l'origine ethnique, à la religion, à l'âge, au handicap ou à l'orientation sexuelle.

Mais si ces mesures préférentielles peuvent être qualifiées de "droits catégoriels", puisqu'elles reviennent à accorder des droits spécifiques à des catégories de personnes, elles ne sont pas pour autant antinomiques avec les droits universels qu'elles contribuent à rendre effectifs : elles tirent les conséquences de ce que l'égalité juridique que consacre la formulation universelle de la règle ne suffit pas à garantir l'égalité réelle.

Un constat analogue a présidé au combat mené pour la "parité » qui suppose de catégoriser les citoyens comme « hommes » ou «femmes », donc de rompre avec le postulat du citoyen abstrait et les présupposés du « modèle républicain ». Il est vrai que certains plaidoyers pour la parité se situent clairement sur un terrain "différentialiste »: c'est le cas lorsqu'on justifie l'objectif d'une représentation égale des hommes et des femmes par l'existence d'une différence irréductible entre les deux sexes qui mériterait d'autant plus d'être prise en compte au niveau de la représentation politique que les femmes auraient des qualités, des centres d'intérêt, des modes de raisonnement qui modifient leur façon de faire de la politique.

Mais la parité peut également se justifier par des arguments de type universaliste dès lors qu'elle vise à permettre aux femmes d'exercer effectivement les droits qui leur sont reconnus théoriquement. Dans cette optique, la parité est un moyen faire advenir concrètement la dimension universelle de la citoyenneté et de mettre fin à l'hypocrisie d'un universalisme abstrait qui s'accommode de la sous-représentation massive des femmes dans les instances politiques.

Un autre domaine où l'on a vu ressurgir les droits catégoriels est celui de la protection sociale. La mise en place, sur une base universaliste, d'un système de sécurité sociale destiné à couvrir «l'ensemble de la population du pays contre l'ensemble des facteurs d'insécurité »a contribué à garantir l'universalité des droits sociaux, rompant ainsi avec le système de l'assistance accordée aux personnes dans le besoin, qui n'était qu'une forme modernisée de l'ancienne charité. Mais la montée des phénomènes d'exclusion conjuguée avec la crise des

\footnotetext{
${ }^{3}$ La Cour de justice des Communautés europénnes a ainsi jugé que constituait une discrimination fondée sur le sexe le fait pour un employeur, utilisant une clause contractuelle qui lui permettait de licencier les travailleurs après un nombre déterminé de semaines d'absence continue, d'avoir licencié une travailleuse enceinte en raison d'absences dues à l'incapacité de travail découlant de son état de grossesse (CJCE, 30 juin 1994, Brown).
} 
systèmes de protection sociale a conduit à la multiplication des prestations ciblées en faveur des plus démunis. Le ciblage, qui revient à reconnaître des «droits catégoriels », rompt assurément avec le modèle universel mis en place après la guerre et qu'on avait progressivement perfectionné. Mais si l'on veut garantir plus efficacement les droits des personnes les plus vulnérables, ne faut-il pas se résoudre à concevoir l'universalité autrement que sous la forme de prestations uniformes pour tous et tenter de combiner les deux systèmes, comme le suggère le Comité des droits économiques, sociaux et culturels ${ }^{4}$ ? Si les États, rappelle-t-il, doivent garantir le droit de tous à la sécurité sociale, sans discrimination aucune, de droit ou de fait, ils doivent aussi être "spécialement attentifs aux individus et aux groupes qui éprouvent des difficultés à exercer ce droit », comme les femmes, les chômeurs, les travailleurs du secteur informel, les travailleurs à domicile, les personnes âgées, les enfants ou encore les groupes minoritaires, les réfugiés, les étrangers ou les détenus.

\section{Les droits catégoriels liées à des appartenances identitaires}

La situation est différente lorsque les droits catégoriels concernent les membres dont l'appartenance à un groupe repose sur une affiliation identitaire : la rupture avec le modèle universaliste est ici incontestable puisqu'il s'agit bien de reconnaître des droits fondés sur une différence revendiquée comme telle et non pas de permettre l'accès à des droits reconnus à tous.

La question se pose néanmoins de savoir si, pour respecter en chaque individu sa part d'altérité, on ne doit pas accepter de prendre en compte des appartenances constitutives des identités individuelles et même faire une place aux identités collectives. Et donc, par voie de conséquence, reconnaître des droits spécifiques aux membres de groupes minoritaires.

La Déclaration des droits des personnes appartenant à des minorités nationales, ethniques, religieuses et linguistiques adoptée en 1992 demande aux États de protéger l'existence et l'identité nationale ou ethnique, culturelle, religieuse ou linguistique des minorités, de prendre des mesures pour que les personnes concernées puissent exercer " intégralement et effectivement » l'ensemble des droits et libertés sans discrimination, mais aussi pour leur permettre « d'exprimer leurs propres particularités et de développer leur culture, leur langue, leurs traditions et leurs coutumes ». Sont donc ici conjugués droits universels et droits spécifiques. Mais en cas de conflit, les droits universels l'emportent sur les droits spécifiques car le droit des minorités de développer leurs traditions et leurs coutumes cède dans le cas où ces pratiques spécifiques « sont contraires aux normes internationales » (art. 4, al. 2).

De même, dans la Déclaration sur les droits des peuples autochtones, adoptée en 2007, on trouve proclamés conjointement le droit à l'égalité et le droit à la différence, des droits universels et des droits spécifiques. L'Assemblée générale reconnaît le droit de tous les peuples " d'être différents, de s'estimer différents et d'être respectés en tant que tels ", elle proclame qu'ils ont des droits collectifs « indispensables à leur existence, à leur bien-être et à leur développement intégral en tant que peuples » : droits culturels, destinés à préserver leur identité, droits de nature politique comme le droit à l'autodétermination, impliquant le droit d'avoir des institutions politiques, juridiques, économiques, sociales et culturelles distinctes, droit aux terres... Mais ici encore la priorité est donnée, en cas de conflit, aux droits universels : la conservation des coutumes et traditions doit se faire « en conformité avec les normes internationales relative aux droits de l'homme » (art. 34), et le dernier article de la Déclaration précise explicitement que, « dans l'exercice des droits énoncés dans la présente Déclaration, les droits de l'Homme et les libertés fondamentales de tous sont respectés » (art. 46).

La revendication par les membres des groupes minoritaires du droit de préserver leur identité heurte de front, au moins en apparence, le postulat universaliste puisqu'elle suppose la

4 Observation générale $n^{\circ} 19$ sur le droit à la sécurité sociale, novembre 2007, $§ 29$ et s. 
reconnaissance de droits spécifiques, qui leur sont réservés. Pourtant, si l'on admet que les hommes ne sont pas seulement des citoyens abstraits mais aussi des individus porteurs d'une histoire et d'une culture singulières, la prise en compte des appartenances ne signifie pas nécessairement l'abandon de l'idéal universaliste : car en occultant les aspirations des groupes minoritaires, on prive les membres de ces groupes de la possibilité d'exercer un certain nombre de droits fondamentaux, notamment d'ordre culturel et politique, pourtant proclamés comme universels. Dans cette perspective, la reconnaissance de droits spécifiques - donc « catégoriels »- peut être considérée comme la condition de l'universalité effective des droits de l'homme et s'inscrire dans une conception de l'universalité moins abstraite, construite sur l'acceptation des différences plutôt que sur leur négation.

Quels enseignements peut-on tirer de la typologie que 1'on vient d'esquisser s'agissant des « droits des femmes »? L'expression renvoie en premier lieu - et avant tout - à l'idée que les femmes doivent avoir les mêmes droits que les hommes, qu'elles doivent pouvoir jouir des droits réputés universels à égalité avec les hommes. Dans cette première acception, les « droits des femmes » ne sauraient à l'évidence être assimilés à des droits « catégoriels ». Les «droits des femmes » peuvent aussi recouvrir, en second lieu, des droits spécifiques : les uns visent à prendre en considération les contraintes directement liées à la gestation, les autres, qualifiées dans les textes de " mesures spécifiques » ou d' " actions positives », à compenser en principe temporairement - les désavantages liés au genre qui, bien au-delà de la " différence » biologique, découlent d'un long passé d'oppression et de relégation dans la sphère domestique. Droits catégoriels ? Oui, certes, dans leur énonciation, mais non dans leur finalité, puisqu'il s'agit bien d'établir les conditions d'une égalité effective, de garantir dans les faits l'universalité des droits que l'égalité juridique n'est pas en mesure d'assurer.

Y a-t-il des «droits des femmes » qui s'apparenteraient à des droits identitaires parce qu'ils ne seraient revendiqués que par et pour les femmes, de façon pérenne et non pas temporaire, en raison d'une différence fondée en nature ou en culture qui en ferait un groupe à part dans la société ? Certains des arguments avancés pour justifier la parité paraissent aller en ce sens : on a ainsi fait valoir que la représentation égale des deux sexes dans les instances politiques était une nécessité en soi parce que l'humanité s'incarne dans des hommes et des femmes ou encore, dans une perspective ouvertement différentialiste, parce qu'il existerait entre les hommes et les femmes une différence irréductible qui s'enracine dans la nature et transcende donc l'évolution historique. Mais cette conception - minoritaire - n'est pas celle qui l'a emporté dans la mise en œuvre de la parité.

En résumé, l'idée que les "droits des femmes » seraient des droits catégoriels antagoniques des droits universels ne résiste pas à une analyse un peu plus fine du contenu et de la finalité de droits qui ne sont catégoriels qu'en apparence. 